\title{
Observation of Real Contact Area Using Laser Microscope with Wide Field of View*
}

\author{
Isami NITTA**, Rintaro EBUCHI**, Yukiko YASUDA*** \\ and Hirotoshi TERAO*** \\ ${ }^{* *}$ Department of Mechanical and Production Engineering, Niigata University, \\ Ikarashi 2-nocho 8050, Nishi-ku, Niigata 950-2181, Japan \\ E-mail: nitta@eng.niigata-u.ac.jp \\ ${ }^{* * *}$ Alps Electric Co., Ltd. HMI Business Unit, Engineering Dept.5 \\ 6-3-36 Nakazato, Furukawa, Osaki 989-6181, Japan
}

\begin{abstract}
In tribology, a contact condition between mating surfaces is very important to reduce friction and raise their lifetime. Direct observation of apparent contact area is necessary for a better understanding of the contact condition. We examined the distribution of real contact area between a platen rubber roller and a glass plate using a laser microscope with wide field of view. Based on these observations, the distribution of real contact area ratio, which is the ratio of real contact area to the apparent contact area at each point, was calculated. Micro-slips over the whole apparent contact area were measured with several torques applied to the platen roller. The relationship between real contact area ratio and micro-slips on the contact area are discussed.
\end{abstract}

Key words: Laser Microscope, Laser Scanning, Confocal, Real Contact Area, Micro-Slip, Rubber Roller

\section{Introduction}

It is very important to examine real contact situations of two solid surfaces in contact because tribological phenomena, such as friction and wear, will not occur evenly over the apparent contact area but will occur at real contact points only. For example, for precise prediction of leakage rates from static metal seals, and contact thermal/electrical resistance at two solid surfaces, it is necessary to determine the dimensions and distributions of the real contact area on the contacting surfaces. In the static metal seal, we have clarified that the leakage rates between a copper gasket and a steel flange can be precisely predicted based on the distribution of real contact area between them ${ }^{(1)}$.

Generally, measurement of real contact area is not easy, and is hardly carried out in practice. The two main reasons why the measurement of real contact area is difficult are as follows. First, one of two solid surfaces in contact is restricted to a transparent material. Second, it takes too much time to observe the whole apparent contact area. With regard to the former problem, we have developed a method for indirect measurement using a thin polymer film. In addition, the real contact area and their distributions between two opaque solids in contact could be measured ${ }^{(2)}$. However, with regard to the latter problem, useful measurement methods have yet to be proposed.

In cases in which one of the mating surfaces is transparent, a contact microscope can be used to measure the real contact area ${ }^{(3)}$. As the contact microscope is within a category of an optical microscope, there is a tradeoff relationship between field of view (FoV) and

*Received 28 Mar., 2011 (No. T2-10-0571)
Japanese Original : Trans. Jpn. Soc. Mech.
Eng., Vol.77, No.775, C (2011),
pp.631-640 (Received 21 July, 2010)
[DOl: 10.1299/jamdsm.5.150]

Copyright $(\underset{0}{ } 2011$ by JSME 
Table 1 Specification of equipment

\begin{tabular}{cc}
\hline Laser source & $\begin{array}{c}\text { Laser diode } \\
(650 \mathrm{~nm})\end{array}$ \\
\hline Field of view $\left[\mathrm{mm}^{2}\right]$ & $10 \times 8$ \\
\hline Resolution $[\mu \mathrm{m}]$ & 2.5 \\
\hline Number of pixels $[\mathrm{pixels}]$ & $20000 \times 16000$ \\
\hline $\begin{array}{c}\text { Speed of observation } \\
{[\text { Lines } / \text { min }]}\end{array}$ & 9000 \\
\hline
\end{tabular}

resolution. Thus, to observe the whole apparent contact area with the contact microscope it is necessary to divide the apparent contact area into many tiny areas that can be covered by the contact microscope and iterate the measurements with the specimen moved step by step. This takes a great deal of effort and time.

Previously, we developed a confocal laser microscope with a wide FoV that can cover a wide area ${ }^{(4)}$. Application of this laser microscope to measurement of the real contact area will allow us to obtain the distributions of the real contact area over the wide apparent contact area in a very short time. In this study, its applicability to measurement of the real contact area was examined by experiment. Rubber specimens, the real contact area of which has hardly been reported, were used in this study. Rubber rollers are used in printers as frictional elements to precisely feed the paper. An understanding of contact conditions between the rubber rollers and the paper is very important for prediction of frictional forces between them and for clarification of micro-slip phenomena. Numerical analyses of contact conditions between the rubber rollers and the paper have been reported ${ }^{(5),(6)}$. In these previous studies, the frictional coefficients between the rollers and paper were assumed to be constant over the whole contact area. However, the frictional coefficients may not be constant but may vary among different points. This must be clarified through experiments involving measurement of the real contact area.

In this study, measurement of the real contact area over the whole apparent contact area was performed using a laser microscope with a wide FoV. The distributions of the real contact area between a rubber disk $5 \mathrm{~mm}$ in diameter and a glass plate were measured using a laser microscope and a conventional contact microscope. The measured results obtained by the two different techniques were compared. Moreover, as an example of wide apparent contact area, the contact surface between the rubber platen roller and the glass plate was observed. The distributions of the micro-slips were obtained using an image correlation technique based on template matching between two images before and after loading

\section{Outline of the Laser Microscope with Wide Field of View}

Figure 1 shows an outline of the laser microscope with wide FoV and the loading system. In this figure, the region within the broken lines indicates the laser microscope with wide FoV, which we developed in a previous study ${ }^{(4)}$. Table 1 shows the specifications of this laser microscope. The laser microscope is constructed based on the principle of confocal microscopy. As a scanning lens, the $\mathrm{f} \theta$ lens is assembled based on a shrink fitter technique ${ }^{(7)}$, the sizes of the laser spots on the focal plane can be kept constant over a wide scanning width. This is why this laser microscope has a wide FoV.

First, a laser beam bundle emitted by a laser diode is made as parallel as possible through a collimating lens. The wavelength of the laser was $650 \mathrm{~nm}$. In addition, a linearly polarized collimated laser beam is transformed into a circularly polarized beam by a quarter wave plate. The laser beam is scanned by a rotating flat mirror at a rotational speed 


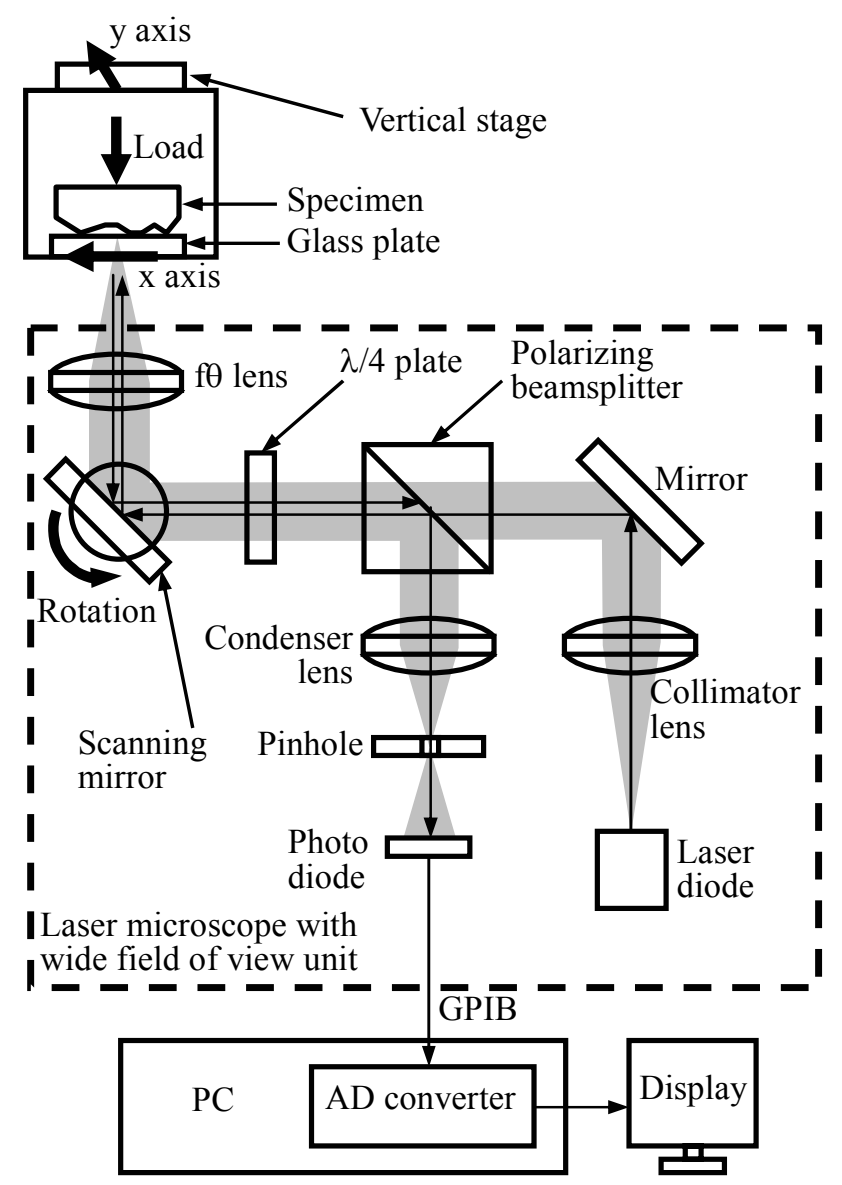

Fig. 1 Outline of observation system

of $9000 \mathrm{rpm}$ and passes through the $\mathrm{f} \theta$ lens unit to focus the laser beam bundle on the focal plane at a laser spot with a diameter of about $4 \mu \mathrm{m}$ over a scanning width of $10 \mathrm{~mm}$. The reflected laser beam becomes polarized at right angles to the outgoing laser beam from the $\mathrm{f} \theta$ lens unit, which allows good separation at the polarizing beam splitter. Finally, the reflected laser beam passes to a photodetector through a pinhole. The intensity of the reflected laser beam is transformed into digital data by a 12-bit $\mathrm{A} / \mathrm{D}$ converter at a conversion rate of $100 \mathrm{MHz}$. The rotating flat mirror scans the laser beam in the horizontal direction (the $\mathrm{x}$ direction), and the specimen attached to the motor-driven stage can be moved in the vertical direction (the y direction) at a constant speed controlled by a microcomputer ${ }^{(4)}$. Thus, the surface of the rubber specimen to be observed can be scanned by the fine laser beam and the image of the rubber surface is produced by arranging the signals of the reflected laser light in the horizontal and vertical directions.

This $\mathrm{f} \theta$ lens unit has a telecentric property such that the outgoing laser beams from the $\mathrm{f} \theta$ lens to the rubber specimen to be inspected are parallel to the optical axis of the $\mathrm{f} \theta$ lens unit. Telecentric lenses yield constant magnification over a range of working distances, virtually eliminating viewing angle error. With telecentric lenses, the image size remains almost unchanged when the object distance changes, provided the object to be inspected remains within the given field depth/telecentric range. In addition, the $\mathrm{f} \theta$ lens unit must be designed such that the image height is proportional to the scan angle, $\theta$, not the tangent of that angle: converting the equiangular motion of the laser beams to the constant speed motion for the scanning operation.

The loading system was constructed to press the rubber specimens against the glass plate $3 \mathrm{~mm}$ thick at a given load, which was measured with a load cell. This loading 
system was fixed to a motor-driven linear stage. Thus, the laser beam can be scanned in the $\mathrm{y}$ direction by this linear stage under the PC control. In the experiments, the contact interface between the rubber and the glass plate was observed from the glass plate side with the laser microscope.

\section{Observation of Real Contact Area}

\subsection{Contact microscope observation}

To verify whether the real contact area can be observed with the laser microscope that we developed, it is necessary to compare the real contact area obtained with the laser microscope to that obtained with the conventional contact microscope. Thus, the real contact area was first observed with the contact microscope. The specimens used were synthetic rubber disks (chloroprene rubber, CR) $1.5 \mathrm{~mm}$ thick, $5 \mathrm{~mm}$ in diameter, with hardness of 50 on shore A scale. The rubber disk was pressed against the right-angle prism at a load ranging from $1 \mathrm{~N}$ to $40 \mathrm{~N}$, and its contact interface was observed with the contact microscope.

Figure 2 shows the real contact area measured with the contact microscope. These images are after thresholding and the black portions in these images represent the real contact area between the rubber and the prism. The thresholding value was determined

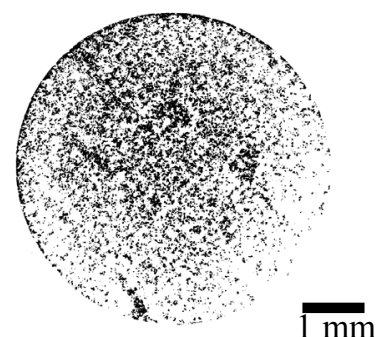

(a) Load $1 \mathrm{~N}$

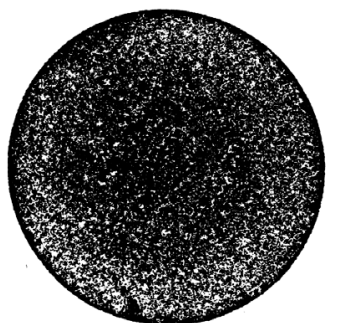

(c) Load $5 \mathrm{~N}$

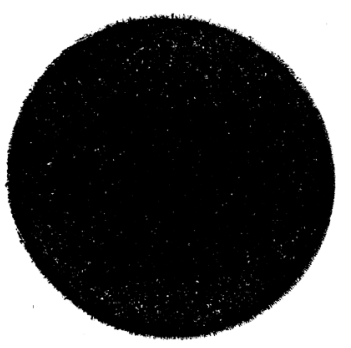

(e) Load $20 \mathrm{~N}$

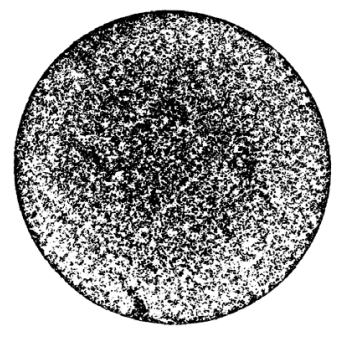

(b) Load $2.5 \mathrm{~N}$

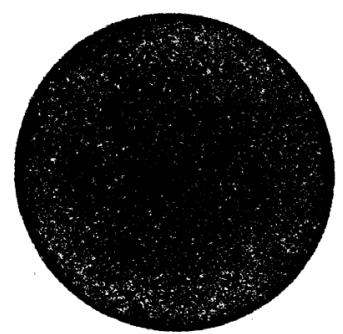

(d) Load $10 \mathrm{~N}$

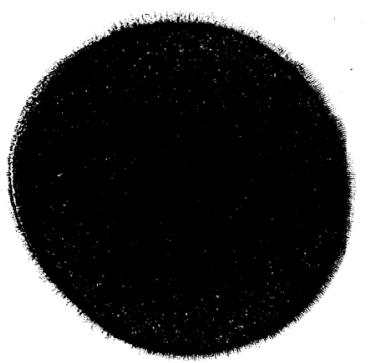

(f) Load $40 \mathrm{~N}$

Fig. 2 Real contact area

(Microscope for real contact area) 
beforehand based on the Hertz contact theory ${ }^{(8)}$. It was confirmed that the real contact area increased with increasing load.

\subsection{Laser microscope observation}

The real contact area was measured with the laser microscope that we developed using the same rubber specimens as described in the section 3.1 above. Figure 3 shows the measured real contact area with the laser microscope. In thresholding, the threshold value was determined by the mode method, finding two peaks (modes) and the local minimum between them. The black portions in these images represent the real contact area between the rubber and the glass plate. Similar to the results obtained with the contact microscope, it was confirmed that the real contact area increases with increasing load. This method is based on two-beam optical interferometry. The real contact area where the interstice between two surfaces makes zero-order dark interference based on optical path difference of reflected light from the lower surface of the rubber and the glass plate. Thus, the real contact area appears dark in these images ${ }^{(9)}$.

\subsection{Comparison of real contact areas obtained by two different methods}

The real contact areas obtained by two different methods were compared as shown in

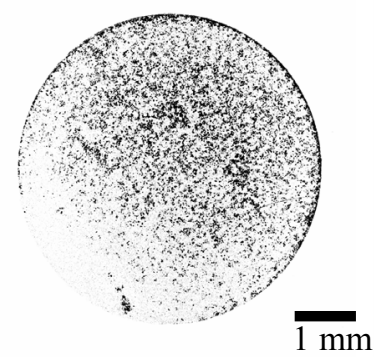

(a) Load $1 \mathrm{~N}$

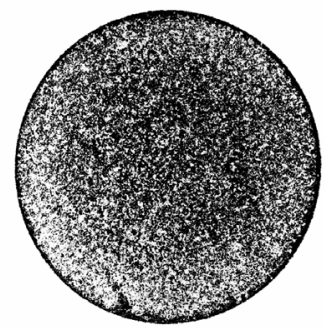

(c) Load $5 \mathrm{~N}$

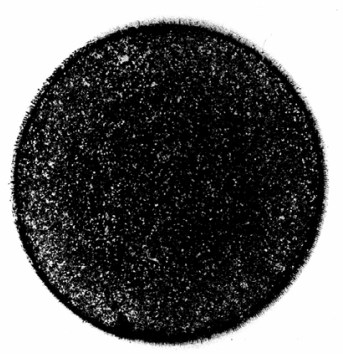

(e) Load $20 \mathrm{~N}$

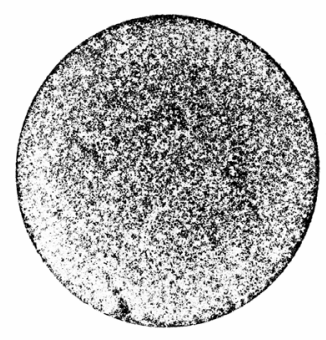

(b) Load $2.5 \mathrm{~N}$

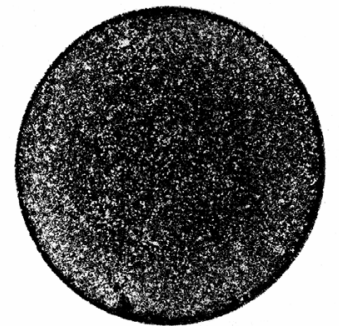

(d) Load $10 \mathrm{~N}$

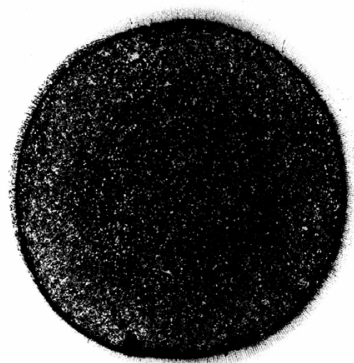

(f) Load $40 \mathrm{~N}$

Fig. 3 Real contact area

(Laser microscope with wide field of view) 
Figs 4(a) and (b). The applied load was $2.5 \mathrm{~N}$. They were similar to each other. The density of the real contact area became relatively high around the periphery and at the center of the apparent contact area. The same portions of (A) in Fig 4(a) and (B) in Fig 4(b) are enlarged to elucidate the real contact area. Figure 4(c) shows an enlarged image of position (A) superimposed on that of position (B). In this figure, white and black regions correspond to non-contact and to contact portions, respectively, as determined by both measurement techniques. Light and dark gray regions indicate the real contact area judged only by contact microscopy or only by laser microscopy, respectively. The size distribution of the real contact area observed with the laser microscope was finer than that observed with the contact microscope. One reason for this result is that the magnitude of the objective lens of the contact microscope is $5 \times$ whereas that of the laser microscope is about $7 \times$ by conversion based on the objective lens of the microscope. Moreover, the laser microscope illuminates only a very small circle area $4 \mu \mathrm{m}$ in diameter at a time, and the real contact is judged based on the reflected laser light from this small area only. On the other hand, the contact microscope illuminates the whole apparent contact area at the same time. The reflected light from a small portion on the rubber specimen surface will be affected and changed slightly by the reflected light on the adjacent portions.
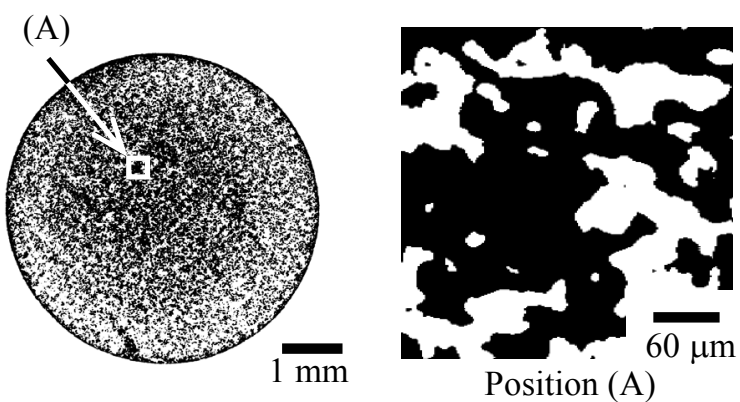

(a) Contact microscope

(B)

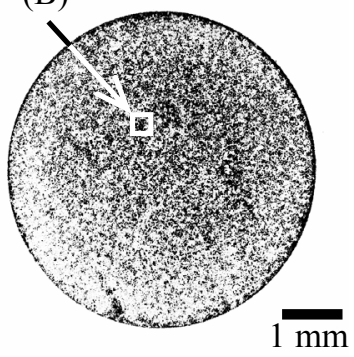

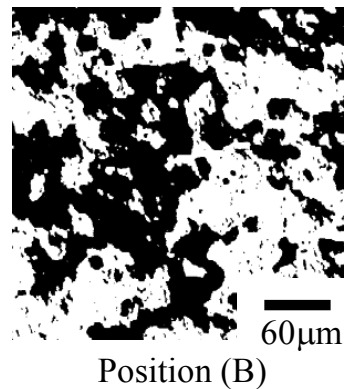

Position (B)

(b) Laser microscope with wide field of view

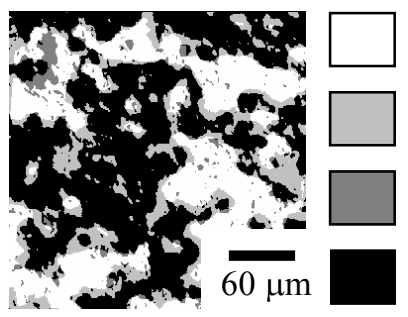

Matching (Contactless)

Contact only (A)

Contact only (B)

Matching (Contact)

(c) Comparison of position (A) and position (B)

Fig. 4 Comparison of real contact areas obtained by two different techniques 


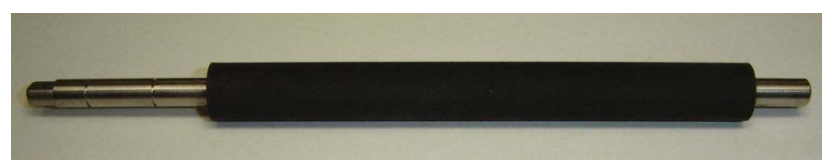

(a) Photograph of rubber platen roller

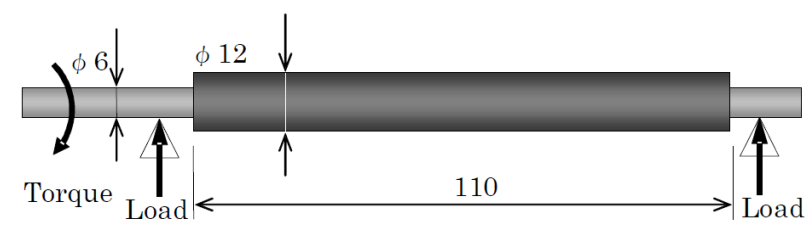

(b) Dimensions of rubber platen roller

Fig. 5 Rubber platen roller

\section{Observation of Rubber Platen Roller}

\subsection{Measurement of distribution of real contact area}

As an example of the observation of the wide apparent contact area, the contact surface between a rubber platen roller and a glass plate was observed. Rubber platen rollers are used in thermal transfer printers. The paper and ink ribbon are sandwiched by the rubber platen roller and the thermal head. The rubber platen roller gives an appropriate load for ink to transfer to the printing paper. Thus, a detailed understanding of the contact situation of the rubber platen roller is very important for high quality printing. Figure 5 shows a photograph of the rubber platen roller used along with a steel shaft. The platen roller has an outer diameter of $12 \mathrm{~mm}$, inner diameter of $6 \mathrm{~mm}$, and length of $110 \mathrm{~mm}$. The diameter of the steel shaft is $6 \mathrm{~mm}$. The normal load was $20 \mathrm{~N}$ applied at each end of the steel shaft, for a total of $40 \mathrm{~N}$. As the FoV of the laser microscope is $10 \mathrm{~mm}, 12$ measurements were needed to cover the whole width of the platen roller.

Figure 6 shows the measured results of the real contact area between the rubber platen roller and the glass plate after thresholding based on the mode methods. The whole images of the contact surfaces cannot be shown here due to space limitations. Thirteen small images measuring $1 \mathrm{~mm} \times 4 \mathrm{~mm}$ were cut out and stored every $5 \mathrm{~mm}$ distance in the shaft direction and relocated as shown in Fig 6 (a). In this figure lateral and vertical correspond to the shaft direction and the circumferential direction of the platen roller, respectively. The contact width decreases to its minimum value at the center of the contact length and expands to maximum values at both ends of $0 \mathrm{~mm}$ and $110 \mathrm{~mm}$ in the shaft direction. Magnified images of parts (A) and (B) in Fig 6(a) are shown in Figs 6(b) and (c), respectively. The distributions of real contact areas are not uniform but show some mesh-like patterns because the surface of the rubber platen roller was finished by grinding and mesh-like patterns of the micro-asperities were produced on the roller surfaces. Based on the images in Fig 6(a), the real contact ratios were calculated over small areas of $250 \mu \mathrm{m}$ $\times 125 \mu \mathrm{m}$ in lateral and vertical directions, respectively, as shown in Fig 7. It can be clearly seen that the real contact area ratios at both ends in the shaft direction are higher than those at the center. This trend in the real contact area ratio may be estimated without measurement. However, the quantitative measurements of the contact widths and the real contact area ratios are very important and necessary for a higher level of printer design. 


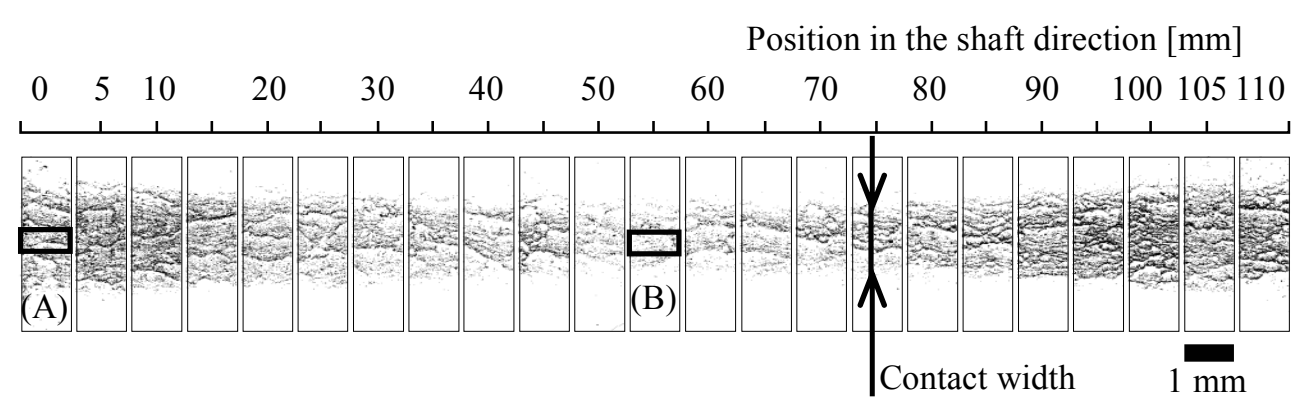

(a) Real contact area of rubber platen roller

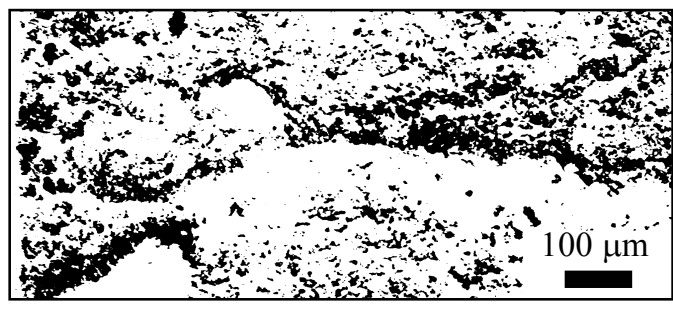

(b) Detail in (A)

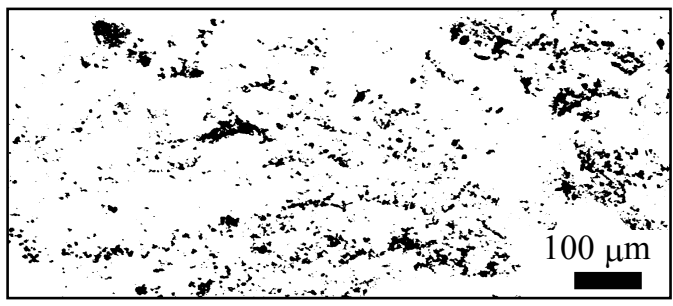

(c) Detail in (B)

Fig. 6 Real contact area (rubber roller)

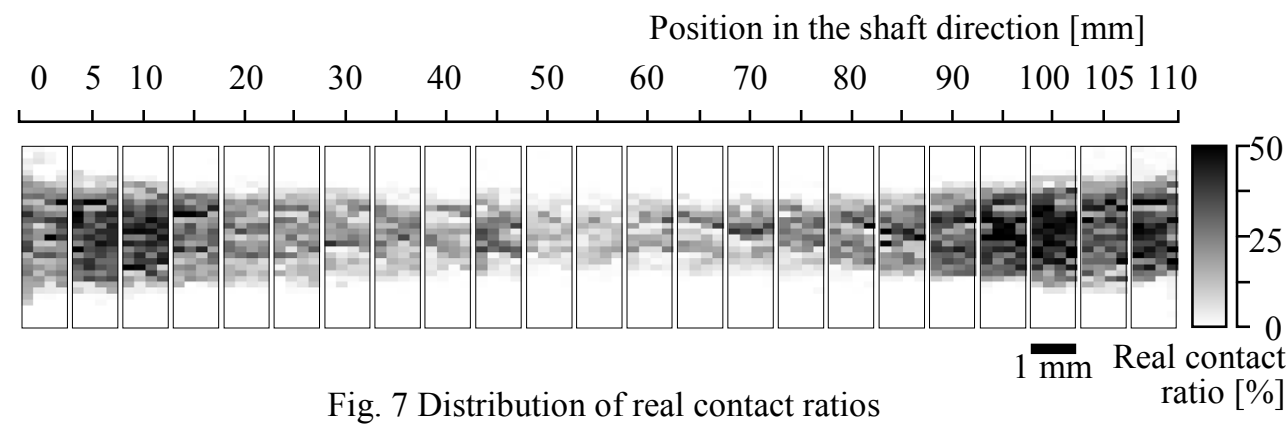

\subsection{Measurements of micro-slips on the contact surfaces}

Using a template matching technique based on the measured images with the laser microscope, micro-slips occurring between the rubber platen roller and the glass plate were analyzed. When the rubber platen roller was pressed against the glass plate at a normal load of $40 \mathrm{~N}$, a torque ranging from 0 to $117.7 \mathrm{~N} \cdot \mathrm{m}$ was applied step by step at the left side of the steel shaft as shown in Fig 5(b). The torque direction was determined such that the real contact points slid downward in the vertical direction in Fig 6(a). We applied the template matching technique to the two images measured before and after application of torque, and calculated the micro-slips corresponding to every template. Template matching is a technique in digital image processing for finding small parts of an image that match a template image ${ }^{(10)}$. In this case the template was made by dividing the contact image before applying the torque into many small parts of the same size. Attempts are made to find the 

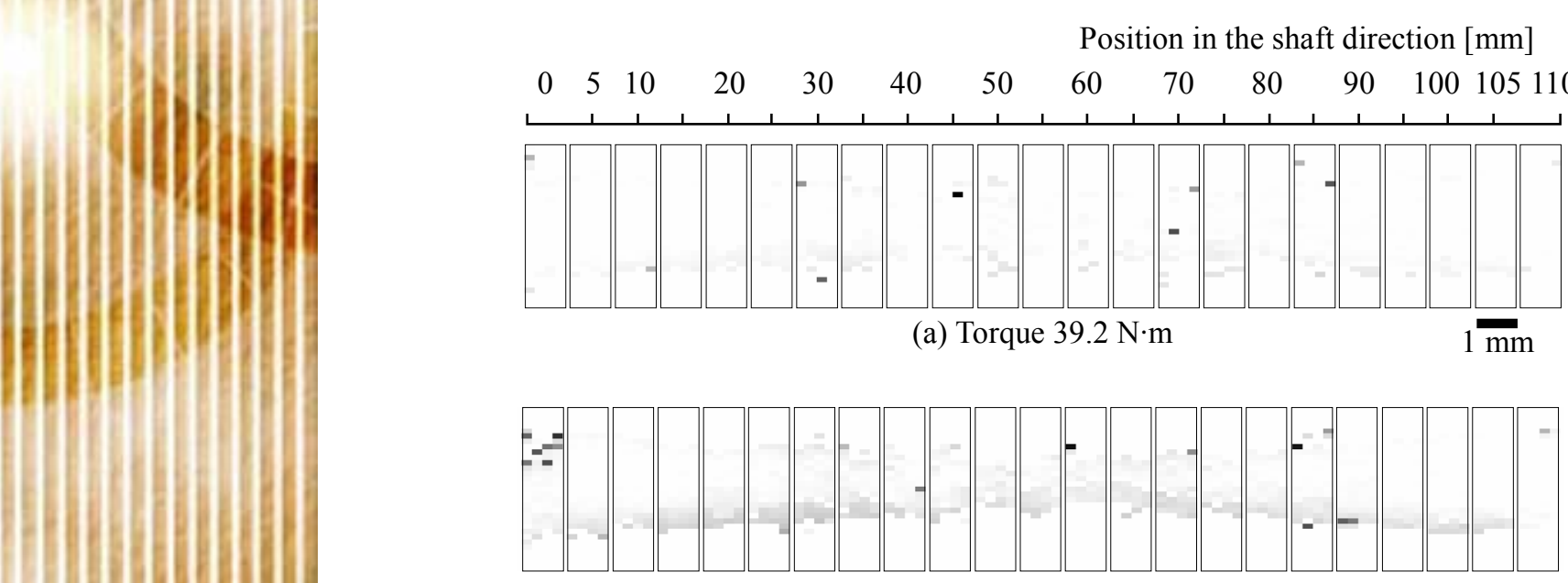

(b) Torque $78.5 \mathrm{~N} \cdot \mathrm{m}$

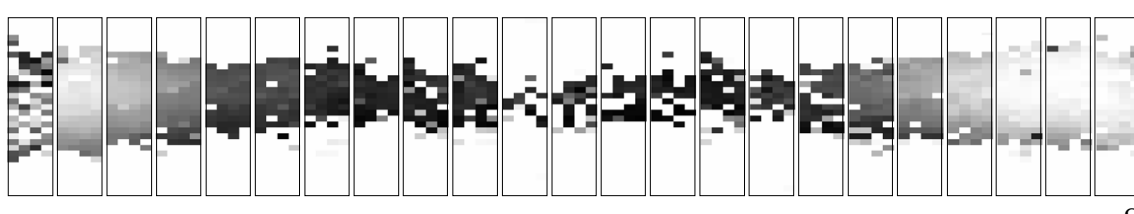

(c) Torque $117.7 \mathrm{~N} \cdot \mathrm{m}$

Fig. 8 Distribution of micro-slips

same patterns as these templates over the contact image after applying the torque. If the template pattern is matched to some extent, i.e., the correlation coefficient reaches the highest value, the movement distance of the template from the original place corresponds to a micro-slip. The size of one template image is $300 \mu \mathrm{m} \times 100 \mu \mathrm{m}$, in the horizontal and vertical directions, respectively. The correlation coefficient was calculated for each downward movement of the template image in the vertical direction by $10 \mu \mathrm{m}$. This procedure was repeated until the movement distance of the template reached $500 \mu \mathrm{m}$. Within the movement distance of $500 \mu \mathrm{m}$, that at which the correlation coefficient reached the highest value was determined as the micro-slip value. Figure 8 shows the distributions of the micro-slips between the rubber platen roller and the glass plate over the apparent contact area calculated in this way. At a low torque of $39.2 \mathrm{~N} \cdot \mathrm{m}$ there was almost no micro-slip across the entire apparent contact area. When the torque increased to $78.5 \mathrm{~N} \cdot \mathrm{m}$, micro-slips of about $30 \mu \mathrm{m}$ begin to occur at the lower periphery of the apparent contact area. In these regions, the surface of the rubber platen roller gradually begins to come apart from the glass plate according to the magnitude of the applied torque. In addition, the elastic deformation by the normal load and the torque will be released and micro-slips will occur. On the other hand, micro-slips occurred at the upper periphery that were smaller than those at the lower periphery. At the highest torque of $17.7 \mathrm{~N} \cdot \mathrm{m}$ the micro-slips spread over the entire apparent contact area. Especially, the micro-slips were larger at the center of the contact area in the shaft direction and smaller at both ends. The micro-slips became larger where the real contact area ratios were smaller, i.e., local contact pressure was lower, based on both Fig 7 and Fig 8 simultaneously.

With this new method for observing wide apparent contact area, the contact situation and the micro-slips of the paper feeding rubber rollers that are usually difficult to observe can be measured and analyzed for the development of a better flexible media handling system. 


\section{Conclusions}

In this study, we developed a new method for measuring the real contact area using a laser microscope with wide FoV. The measured distributions of the real contact area were compared with those measured with the conventional contact microscope. Moreover, the wider contact surface between the rubber platen roller and the glass plate was measured with this new technique. Based on the results of this study, the following conclusions were obtained.

(1) The distributions of the real contact area measured with the laser microscope with wide FoV were similar to those measured with the conventional contact microscope.

(2) The wide contact surfaces of $110 \mathrm{~mm} \times 4 \mathrm{~mm}$ between the rubber platen roller and the glass plate were observed with this new technique. It was verified that the distributions of the real contact area can be measured in a shorter time compared with the conventional contact microscope.

(3) The distributions of micro-slips over the apparent contact area were calculated based on the template matching method. The micro-slips occurred easily where the real contact area ratios showed lower values.

\section{References}

[1] Nitta, I. and Matsuzaki Y., Experimental Study of the Performance of Static Seals Based on Measurements of Real Contact Area Using Thin Polycarbonate Films, Transactions of the ASME, Journal of Tribology, Vol.132, No.2 (2010), pp.22202-1 - 22202-7.

[2] Nitta, I., Measurements of Real Contact Areas Using PET Films (Thickness, $0.9 \mu \mathrm{m}$ ), Wear, Vol.181, No.183 (1995), pp.844-849.

[3] Sakurai, Y. and Takeishi, H., Deformation of a Steel Ball and an Optical Flat in Contact, Journal of the Japan Society of Precision Engineering, Vol.52, No.11 (1986), pp.1941-1946.

[4] Nitta, I., Kanno, A., Okamoto, M. and Nagaoka, Y., Study of a New Type of Laser Microscope with Wide Field of View Using a Shrink Fitter, Journal of the Japan Society of Precision Engineering, Vol.73, No.11 (2007), pp.1226-1232.

[5] Tomimatsu, M. Yamaura, H. and Ono, K., Analysis of Paper Transportation Characteristics of a Long Rubber-layerd Roller and a Steel Roller, Transactions of the Japan Society of Mechanical Engineers, Series C, Vol.71, No.712 (2005), pp.3525-3533.

[6] Okamoto N., Ohtani K., Misawa K. and Yoshida K., Study on Velocity Characteristics and Mechanics of Paper Feeding with Rubber-Covered Roller Drive, Transactions of the Japan Society of Mechanical Engineers, Series C, Vol.67, No.654 (2001), pp.475-482.

[7] Nitta, I., Kanno, A., Karita, N., Komata, K. and Shirai K., Ultra-Precision Method of Joining Optical Lenses Using a Shrink Fitter, Journal of Japanese Society of Tribologists, Vol.44, No.2 (1999), pp.128-134.

[8] Nitta, I., Morohashi, A., Measurements of Distributions of Real Areas Using Thin PET Films, Journal of the Japan Society of Precision Engineering, Vol.67, No.10, (2001), pp.1615-1620.

[9] Eguchi, M., Shibamiya, T. and Yamamoto, T., Measurement of real contact area and analysis of stick/slip region, Tribology International, Vol.42, No.11-12, (2009), pp.1781-1791.

[10] Sakai, K., Manual of digital image processing using Visual Basic \& Visual C++, CQ Publication Corporation (2002), pp.127-129. 